\title{
IIWM- An Invertible Image Watermarking
}

\author{
J Sasi Kiran \\ Professor \& Head \\ Dept. of CSE \\ VVIT, Chevella
}

\author{
PVRD Prasada Rao \\ Professor, Dept. of CSE \\ PVPSIT, Vijayawada
}

\author{
G.Charles Babu \\ Professor, Dept. of CSE \\ VVIT,Chevella
}

\author{
G Ravi Kumar \\ Assoc.Prof \& Head \\ Dept. of MCA \\ VVIT,Chevella
}

\author{
Dr A Yesu Babu \\ Professor \& Head \\ Dept. of CSE \\ CRR Engg College, Eluru
}

\begin{abstract}
In digital watermarking, an invisible signal referred as a watermark is embedded into multimedia data for various purposes such as copyright protection, fingerprinting, authentication etc. For applications where the availability of original data is essential, irreversible degradation of the original data is not acceptable and incurred distortions need to be removed. Examples of such applications include multimedia archives, military image processing, and medical image processing for electronic patient records (EPRs).

High capacity watermarking is proposed in the paper and implemented using integer to integer wavelet transform. The proposed scheme divides an input image into non-overlapping blocks and embeds a watermark into the high frequency wavelet coefficients of each block. The conditions to avoid both underflow and overflow in the spatial domain are derived for an arbitrary wavelet and block size. The experimental results show that the implemented scheme achieves higher embedding capacity while maintaining distortion at a lower level than the existing invertible watermarking schemes.
\end{abstract}

Keywords: - Invertible Watermarking, Wavelet Transform, Watermark embedding and extraction.

\section{INTRODUCTION}

In multimedia archives, a content provider may not want the original content to be distorted even though the distortion is imperceptible to most users, and it may be too costly in terms of storage space to store both the original and the watermarked versions. In military image processing, images are gathered at a very high cost and are usually subjected to further processing such as extreme magnification. Any distortion may hinder accurate analysis of the image.

In medical image processing, any modification to the original image may affect a doctor's diagnosis and lead to legal problems. Any complications that can occur when using a conventional watermarking scheme in the applications listed above can be resolved by using the invertible (lossless, reversible, erasable, etc) watermarking scheme. Although the embedding distortion is inevitable even in invertible watermarking, it can be removed, and the original data can be reconstructed from the watermarked version. Another advantage of the invertible watermarking is that the access to the original content can be controlled. In a conventional watermarking scenario, no one has access to the original content since the distortion due to the embedding of the watermark is not erasable. When the watermark is embedded in a reversible manner, an authorized person can access the original content by erasing the watermark, while the watermarked content is available to everyone.

When the original content can be recovered from the watermarked content, the watermarking scheme is said to have the invertibility (reversibility) property.

The watermark that they embedded into an image was completely invisible but could be removed since it was embedded in a reversible manner. Fridrich et al. extracted a vector which represented specific characteristics of pixel groups, compressed it without any loss, and embedded the watermark bits by appending it to the compressed vector. Tian applied integer Haar wavelet transform to an image and embedded the watermark into high-frequency coefficients by difference expansion (DE).

The first watermarking scheme that was introduced works directly in the spatial domain. By some image analysis operations (e.g. Edge detection), it is possible to get perceptual information about the image, which is then used to embed a watermarking key, directly in the intensity values of predetermined regions of the image. Those pretty simple techniques provide a simple and effective way for embedding an invisible watermark into an original image but don't show robustness to common image alterations.

Another way to produce high quality watermarked image is by first transforming the original image into the frequency domain by the use of Fourier, Discrete Cosine or Wavelet transforms for example. With this technique, the marks are not added to the intensities of the image but to the values of its transform coefficients. Then inverse transforming the marked coefficients forms the watermarked image. The use of frequency based transforms allows the direct understanding of the content of the image; therefore, characteristics of the human visual system (HVS) can be taken into account more easily when it is time to decide the intensity and position of the watermarks to be applied to a given image.

\section{INVERTIBLE INTEGER-TO- INTEGER WAVELET TRANSFORMS}

\section{A. Conventioal Approach}

Conventional wavelet transform is not applicable to the reversible watermarking scheme since it does not guarantee 
the reversibility. For example, suppose that an image block consisting of integer-valued pixels is transformed into a wavelet domain using a floating-point wavelet transform. If the values of the wavelet coefficients are changed during the watermark embedding, the corresponding watermarked image block is no longer guaranteed to have integer values. Any truncation of the floating-point values of the pixels may result in a loss of information and may ultimately lead to the failure of the reversible watermarking systems, that is, the original image can not be reconstructed from the watermarked image. Furthermore, conventional wavelet transform is in practice implemented as a floating-point transform followed by a truncation or rounding since it is impossible to represent transform coefficients in their full accuracy information can potentially be lost through forward and inverse transforms. Embedding, the corresponding watermarked image block is no longer guaranteed to have integer values. Maintaining the Integrity of the Specifications.

To avoid this problem, an invertible integer-tointeger wavelet transform based on lifting is used in the proposed scheme. It maps integers to integers and does not cause any loss of information through forward and inverse transforms. According to, every wavelet or subband transform associated with finite length filters can be obtained as the Lazy wavelet followed by a finite number of primal and dual lifting steps and a scaling (the Lazy wavelet essentially splits the signal into its even and odd indexed samples). By combining the lifting constructions with rounding-off in a reversible way, a wavelet transform that maps integers to integers can be obtained. For example, the integer-to-integer wavelet transform that approximates Le Gall 5/3 filters is given by,

$$
\begin{aligned}
& y(2 n+1)=x(2 n+1)-((x(2 n)+x(2 n+2) / 2) \\
& y(2 n)=x(2 n)+((y(2 n-1)+y(2 n+1)+2)) / 4)
\end{aligned}
$$

Where $\mathrm{x}$ is the extended input signal, $\mathrm{y}$ is the output signal. To make transforms nonexpansive, symmetric extension compatible with invertible integer-to-integer wavelet transform is used.

\section{PREDICTION OF UNDERFLOW AND OVERFLOW}

In the proposed scheme, a watermark is embedded into the wavelet coefficients using either the LSB-substitution or the bit-shifting. With the LSB-substitution technique, the watermark is embedded by replacing the LSB of the selected wavelet coefficient with the watermark bit as follows:

$$
c^{w}=2 x(c / 2)+w
$$

Where $c, c^{w}$, and $w$ are the original and the watermarked wavelet coefficients and the watermark bit, respectively.

Where $w=2^{0} . w_{0}+2^{l} \cdot w_{1}+\ldots \ldots+2^{p-1} \cdot w_{p-l}$ and $\left.w_{0}, w_{1}, \ldots, w_{p-1}\right\}$ is a set of $\mathrm{p}$ watermark bits. Later we will find out that the value of $p$ is adaptively determined for each bit-shiftable block so that the perceptual distortion of the watermarked image is minimized.
In the proposed watermarking scheme, integer-to-integer wavelet transforms are used to achieve the reversibility. Since integer-to-integer wavelet transforms involve the truncations of wavelet coefficients during the lifting steps, round-off error is inevitable

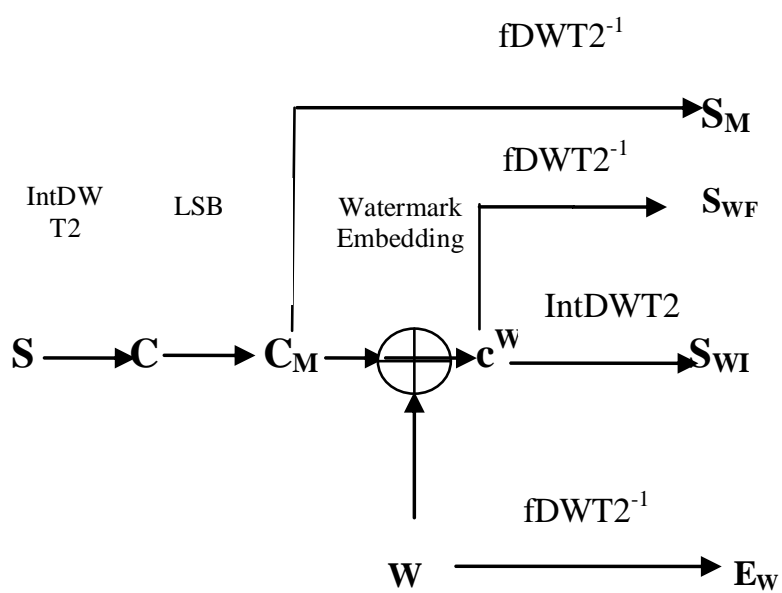

Fig 1: Forward and inverse wavelet transform, and watermark embedding.

When the watermark is embedded in the wavelet domain using either LSB-substitution, underflow or overflow can occur in the spatial domain. That is, the pixel values obtained from the watermarked wavelet coefficients can either be smaller than the minimum pixel value $s_{\text {min }}$ (e.g. $s_{\min }=0$ for 8-bit gray-scale image) or be greater than the maximum value $s_{\max }$ (e.g. $s_{\max }=255$ for 8-bit grayscale image). Since the reversibility is lost when underflow or overflow occurs, it must be predicted prior to the watermark embedding. In this paper, underflow and overflow are predicted by identifying the LSB-changeable image blocks.

\section{Iii.1. Tiling}

The term 'tiling' refers to the partition of the original (source) image into rectangular nonoverlapping blocks (tiles), which are compressed independently, as though they were entirely distinct images. All operations, including component mixing, wavelet transform, quantization and entropy coding are performed independently on the image tiles. Tiling reduces memory requirements and since they are also reconstructed independently, they can be used for decoding specific parts of the image instead of the whole image. All tiles have exactly the same dimensions, except maybe those at the right and lower boundary of the image. Arbitrary tile sizes are allowed, up to and including the entire image (i.e. the whole image is regarded as one tile).

Components with different sub-sampling factors are tiled with respect to a high-resolution grid, which ensures spatial consistency on the resulting tile components.

\section{A. Derivation of Condition to Avoid Underflow and Overflow}

In this subsection, the condition to avoid underflow and overflow is derived. The block-wise embedding using wavelet transforms and the notations that will be henceforth used are described in Fig. 1. First, an $M x N$ pixel block $\mathrm{S}$ is transformed into a block of $M x N$ wavelet coefficients $C$ using the 2-dimensional nonexpansive 
integer-to-integer wavelet transform, IntDWT2(.). Next, a block of modified wavelet coefficients $\mathrm{C}_{M}$ is obtained either by setting the LSBs of the chosen wavelet coefficients to zero or by applying bit-shifting to the chosen coefficients in $\mathrm{C}$. The modified pixel block $\mathrm{S}_{M}$ is obtained by applying the 2-dimensional inverse floating point (fDWT2 $\left.2^{-1}().\right)$ wavelet transform to $\mathrm{CM}$. By adding a watermark bit block $\mathrm{W}$ to $\mathrm{CM}$, a block of watermarked wavelet coefficients $C W$ is obtained. Then, $S_{W F}$ and $S_{W I}$ are obtained by applying (fDWT2 $\left.2^{-1}().\right)$ and $\operatorname{IntDWT} 2^{-1}$ (.) to $\mathrm{CW}$, respectively. The embedding error $\mathrm{EW}$ is obtained by applying (fDWT2- $\left.2^{-1}().\right)$ to W. Henceforth, $\mathrm{A}(m, n)$ represents an element of the block $A$ in the $m^{\text {th }}$ row and the $n t^{h}$ column.

When a floating-point wavelet transform is used, underflow and overflow that can occur as a result of embedding a watermark in the wavelet domain can be easily predicted by exploiting the linearity of the transform. Then the watermarked block $S_{W F}$ in Fig. 1.

\section{PROPOSED INVERTIBLE EMBEDDING ALGORITHM}

Given an $X x Y$ original image to be watermarked, a set of message bits $B m$ to be embedded, block size $(M \times N)$, wavelet to be used for forward and inverse transforms.

\section{A. Performance for Natural Images with Various Block Sizes.}

The proposed scheme is applied to various natural images,F-16 (Airplane), Lena, Barbara, Peppers, Fishing boat, and Baboon (Mandrill). In this experiment, the block size was set to $8 \times 8$. The proposed scheme achieves high embedding capacity with low distortion. However the capacity-distortion performance depends on the characteristic of each image.

In the proposed scheme, the block size can be easily adjusted to the specific requirements of an application. The performance of the proposed scheme varies with different block sizes: $4 x 4,8 x 8,16 x 16$, and $32 \times 32$, the performance of the proposed scheme is degraded when the block size is too small $(4 x 4)$ or too large $(32 x$ 32 ). The smaller the block size, the larger the amount of the side information becomes. The performance degrades when the block size is too small. When the block size is large, the amount of the side information that needs to be embedded is very small. However, block size that is too large makes the adaptive embedding less useful especially for high embedding capacity, and this degrades the performance of the proposed scheme.
The following snapshots represent an Lena image, Watermarked content image and Watermarked bit pattern are used.

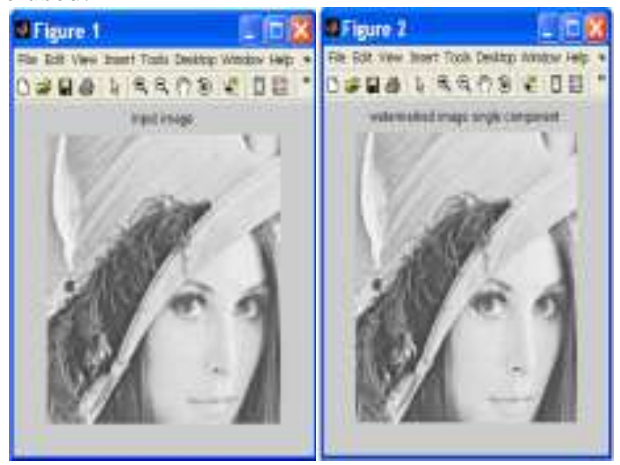

Watermark bit pattern

[1,1,1,0,0,0,0,1,0,1,1,1,1,1,0,0,0,0,1,0,1,1,1,1,0,1,0,0,1,0,1,0]

\section{CONCLUSION}

In this paper, a invertible image watermarking scheme based on integer-to-integer wavelet transforms is proposed. In the proposed scheme, an input image is divided into a number of blocks, and a watermark is embedded into the high-frequency wavelet coefficients of each block via LSBsubstitution. The original image can be exactly reconstructed at the decoder since the side information for achieving the reversibility is also embedded in the image while avoiding the underflow and overflow. Experimental results show that the proposed scheme achieves higher embedding capacity at a lower distortion than other existing invertible schemes. Further to this work, improved invertible watermarking with high capacity with encrypted watermark shall be done.

\section{REFERENCES}

[1] JPEG2000 STILL IMAGE CODING SYSTEM AN OVERVIEW BY Charilaos Christopoulos1 Senior Member, IEEE, Athanassios Skodras2 Senior Member, IEEE, and Touradj Ebrahimi3 Member, IEEE.

[2] R. C. Gonzalez and Richard E. Woods, Digital Image Processing, $2^{\text {nd }}$ Edition, Prentice Hall, 2002.

[3] Sunil Lee (student member IEEE), Chang D. Yoo (Member IEEE) and Ton Kalker (Fellow IEEE)"Reversible Image watermarking based on integer to integer wavelet transform."

[4] USC-SIPI Image Database [Online]. Available. 